\title{
A Mathematical Model of Measles with Vaccination and Two Phases of Infectiousness
}

\author{
Ochoche J. M. and Gweryina R. I. \\ University Of Agriculture, P.M.B 2373, Makurdi, Nigeria \\ jeffreycyborg@gmail.com
}

\begin{abstract}
Despite the availability of the measles vaccine since 1963, the infectious disease is still endemic in many parts of the world including developing and developed nations. It was eliminated from the U.S. in 2000 but due to importation secondary transmissions has been occurring since 2008. With a greater than $90 \%$ attack rate measles is not only a health but also an economic problem as it spread very quick leading to a lot of persons been hospitalized or seeking medical help. The disease is endemic in Nigeria with an annual incidence rate of 18.2 per 100, 000 children and a case fatality rate of $1.2 \%$ as at 2008. The disease is one of the leading causes of child mortality worldwide. Its prevalence and severity in Nigeria might hamper the fourth millennium development goal by 2015 (To reduce by two thirds, between 1990 and 2015, the under five mortality rate).

In this paper we proposed a mathematical model measles incorporating vaccination as a control strategy and capturing the two phases of infectiousness (i.e asymptomatic infectives and symptomatic infectives). We found the basic reproduction number using the next generation approach and proved that our system of equations is locally asymptotically stable if $R_{0}<1$ which means that the disease can be eradicated under such condition in finite time. Furthermore we note that in the presence of vaccination $R_{0}$ approaches 0 as $p$, the proportion of those vaccinated approaches 1. We reiterated the already known fact that close to 100\% (at least 94\%) vaccination of susceptible is required for eradication of the disease and suggests that the government should make the vaccination compulsory in other to achieve the herd immunity. We also used LHS/PRCC to support this claim.
\end{abstract}

Keywords: Basic Reproduction Number, Herd Immunity, Measles, Sensitivity analysis, Vaccination.

\section{Introduction}

Measles is not only health problem but also an economic problem of mankind as outbreaks usually lead to enormous expenditure on healthcare. A 2002 to 2003 outbreak of measles in Italy, which led to the hospitalizations of more than 5,000 people, had a combined estimated cost between 17.6 million Euros and 22.0 million Euros. While a 2008 outbreak of measles in San Diego, California cost $\$ 177,000$, or $\$ 10,376$ per case[1]. The human race has suffered many kinds of infectious diseases such as leprosy, chicken pox, small pox, cholera, malaria, polio, H5N1, SARS, measles and HIV/AIDS. Some of these diseases H5N1are promptly brought under control mostly as a result of behavioral change of the susceptible while others like polio and small pox has been eradicated or at least eliminated in most part of the world as a result of control and preventive measures championed by bodies like WHO, UNICEF and government agencies.Some diseases like small pox and measles are vaccine preventable hence eradication efforts are geared towards vaccination ofsusceptibles. These efforts have paid off in the case of small pox which has been eradicated. However, unlike small pox, the eradication and even elimination of measles still remain elusive in many parts of the world. Some fifty years ago, when the vaccine was made available it was thought that measles will soon become a disease of the past since it shares many features with small pox which has been eliminated. Some the features include (1) a characteristic rash with seasonal occurrence, (2) no vector or animal reservoir with transmissible latent virus , (3) only one serotype and (4) availability of an effective vaccine [2]. Why then has measles not been eradicated? This is the question that the present paper attempted to answer.

Measles, one of the most contagious vaccine preventable diseases in humans is caused by the measles virus, a member of the genus Morbillivirus of the family Paramyxoviridae.Symptoms of measles include fever, runny nose, drowsiness, irritability and red eyes. Small white spots can appear on the inside of the mouth and throat. Complications include diarrhea, pneumonia and infections of the brain. In developed countries 2 to 3 cases per 1,000 result in death. This increases to 3 to 5 cases per 1,000 in developing countries [3].In 1980, before widespread vaccination, measles caused an estimated 2.6 million deaths each year [4]. In 1989 the WHO Expanded Programme on Immunization estimated that 1.5 million children died annually from measles or its complications. Measles is estimated to have caused 614,000 deaths in 2002 [5] while in 2003 worldwide death toll as a result of measles was up to 530,000 [6]. Global death toll as a result of measles and itscomplications was 158,000 as at $2011[4]$. 
Although measles can be contracted by persons of any age, it is prevalent among children aged 5 years and below. Unvaccinated pregnant women are at high risk of complications if they contract the disease. Measles is highly contagious such that greater than $90 \%$ of unimmunized persons who come in contact with the virus get the disease. The virus is spread when an infected person coughs, sneezes or breaths, it remains active in the air or an infected surface for up to two hours. There is no treatment for measles; however, most people recover from the disease. Natural infection results in lifelong immunity.

Compared with levels in 2000, the global goal was to reduce mortality due to measles by $90 \%$ by 2010 . This goal was not reached [3]. The failure of this goal can be attributed to the extremely high transmission potential. Measles has a basic reproduction number of the order 6 to 45 which implies that the average number of secondary infection generated by a single infective in a susceptible population lies between 6 and 45 [7]. Indeed high immunization coverage is required to meet such global goals.In April 2012 the Measles and Rubella initiative launched a new Global Measles and Rubella Strategic Plan which covers the period 2012-2020. The Plan includes new global goals for 2015 and 2020 as follows:By the end of 2015 to (1) reduce global measles deaths by at least 95\% compared with 2000 levels and (2) achieve regional measles and rubella/congenital rubella syndrome elimination goals [4].

Childhood diseases like measles are the most dangerous in developing countries as they have the most significant impact on mortality rate and affect childhood learning, and therefore ultimately on labor productivity [8]. Most infectious diseases are as a direct result of poverty as they are found disproportionately among the poor, who often lack the resources to prevent such disease transmission [9]. Infectious diseases like cholera and malaria are no longer problems of developed countries. With heightened hygiene, proper wastes and environmental management these diseases have been eliminated from developed countries. Measles however remain a global menace as it is not only a problem of developing but also developed nations. Although the World Health Organization (WHO) and its member states across the world have aimed to eliminate measles, the elimination has been so far fully successful only in the North American region[7]. Outside of the Americas, measles activity remains high. As of October 2011, there were over 26,000 cases in the WHO European Region with the highest number reported in France (more than 14000 cases) [3].

Measlesis a vaccine preventable disease; the current practice in most part of the world especially in developed countries is that children aged 12 to 15 months are given a dose of the MMR (Measles, Mumps and Rubella) vaccine. In practice, even vaccinated individual may still be susceptible if vaccination failure occurred or their vaccine - induced immunity waned. Children aged $4-5$ years are therefore given a booster dose [2,7]. In this paper, we modeled the transmission dynamics of measles in the presence of vaccination and investigated itsrole in the dynamics of the disease.

\section{Model Formulation}

We follow the $\mathrm{S}-\mathrm{I}-\mathrm{R}$ modeling approach. The total population is hence partitioned into Susceptible, Infectious and Recovered compartments. People infected with measles are usually contagious from 4 days before until 4 days after the onset of signs or symptoms $[3,10]$. Many people who transmit the disease tend to do so before they are aware that they have the disease. We captured these two phases of infectiousness i.e. transmission of the disease just before symptoms appear and transmissionof the disease after appearance of symptoms in compartmentsXandY respectively. $\beta$ is the probability of infection upon contact. We used mass action as the transmission incidence for $\mathrm{X}$ and we used a saturated incidence for $\mathrm{Y}$. This we justified by the fact that before the symptoms appear (X), there is no inhibition for disease transmission and the asymptomatic infective mix freely with susceptibles, hence the concept of mass action apply. However after the onset of symptoms, susceptibles change their behavior towards the infective and some infectives are placed in negative pressure rooms these constitute inhibiting factors hence the saturation incidence for $\mathrm{Y}$. $0<\alpha<1$ is the parameter that measures the inhibitory effect. The variables and parameters of the model are defined below:

S Susceptible population

$\mathrm{X} \quad$ Infectives at the first phase of infectiousness (Asymptomatic)

$\mathrm{Y}$ Infectives at the second phase of infectiousness (Symptomatic)

$\mathrm{R} \quad$ Recovered individuals

$\mathrm{N} \quad$ Total human population

$\beta \quad$ Probability of infection upon contact

B Birth rate

$\mathrm{P} \quad$ Individuals successfully vaccinated against the disease

$\mu \quad$ Natural death rate

$\theta \quad$ Rate at which the asymptomatically infectious become

symptomatic

$\gamma$

Recovery rate 


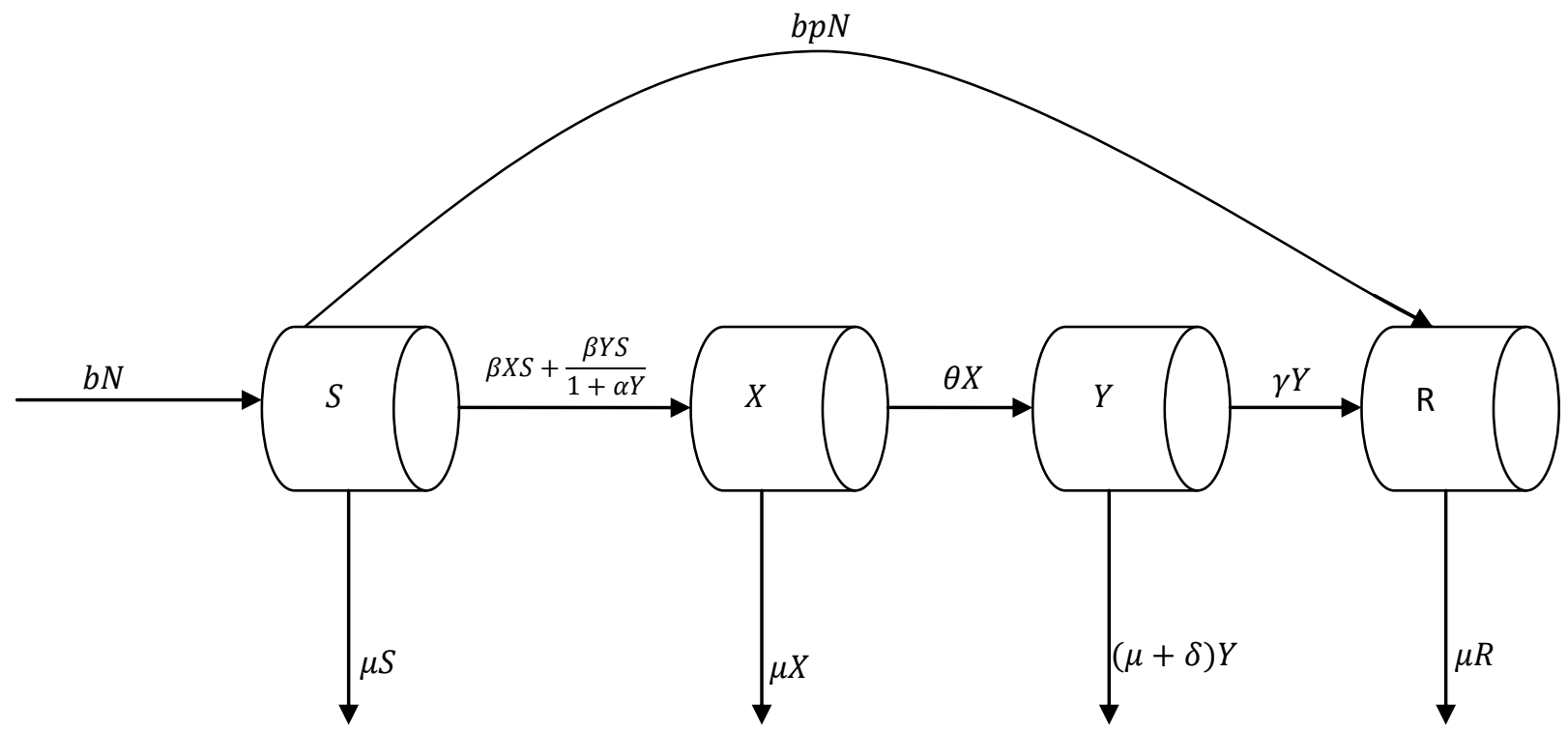

Fig. 1: Flow diagram of the model.

Following the flow diagram the model equations are presented thus;

$\frac{d S}{d t}=b(1-p) N-\beta X S-\frac{\beta Y S}{1+\alpha Y}-\mu S$

$\frac{d X}{d t}=\beta X S+\frac{\beta Y S}{1+\alpha Y}-(\theta+\mu) X$

$\frac{d Y}{d t}=\theta X-(\delta+\gamma+\mu) Y$

$\frac{d R}{d t}=\gamma Y+b p N-\mu R$

Since the variable R those not appear in the system (1), in subsequent analysis we shall consider on the system $\frac{d S}{d t}=b(1-p) N-\beta X S-\frac{\beta Y S}{1+\alpha Y}-\mu S(2)$

$\frac{d X}{d t}=\beta X S+\frac{\beta Y S}{1+\alpha Y}-(\theta+\mu) X$

$\frac{d Y}{d t}=\theta X-(\delta+\gamma+\mu) Y$

Adding the equations of the system (2), we have

$\frac{d S}{d t}+\frac{d X}{d t}+\frac{d Y}{d t}=b(1-p) N-\mu(S+X+Y)-(\delta+\gamma) Y$

$\leq b(1-p) N-\mu(S+X+Y)$

Hence,

$\limsup _{t \rightarrow \infty}(S+X+Y) \leq \frac{b(1-p) N}{\mu}$

Thus the considered region for the system (2) is

$\Gamma=\left\{(S, X, Y): S+X+Y \leq \frac{b(1-p) N}{\mu}, S>0, X \geq 0, Y \geq 0\right\}$.

The vector field points into the interior of $\Gamma$ on the part of its boundary when $S+X+Y=\frac{b(1-p) N}{\mu}$.

Therefore,

$S(t)+X(t)+Y(t)<\frac{b(1-p) N}{\mu}$,For $t>0$.

Hence $\Gamma$ is positively invariant.

\subsection{BASIC REPRODUCTION NUMBER}

Epidemiologists have always been interested in finding the basic reproduction number of an emerging disease because this threshold parameter can tell whether a disease will die out or persist in a population. Denoted by $\mathrm{R}_{0}$, this parameter is arguably the most important quantity in infectious disease epidemiology. It is defined as the average number of new cases (infections) produced by a single infective when introduced into a susceptible population. It is one of the first quantities estimated for emerging infectious diseases in outbreak situations [11].It is a key epidemiological quantity, because it determines the size and duration of epidemicsand 
is an important factor in determining targets for vaccination coverage [5]. The basic reproduction number is sought afterprincipally because:

1. If $\mathrm{R}_{0}<1$, then throughout the infectious period, each infective will produce less than one new infective on the average. This in turn implies that the disease will die out as the DFE is stable.

2. If $\mathrm{R}_{0}>1$, then throughout the infectious period, each infective will produce more than one new infective on the average. This in turn implies that the disease will persist as the DFE is unstable. In other words, there will be an outbreak.

If $\mathrm{R}_{0}$ can be determined, then the transmission parameters which will force $\mathrm{R}_{0}$ to be less than or greater than 1 can easily be identified and control measures effectively designed.

Next, we shall find the basic reproduction number of the system (2) using the next generation method [12].

The disease free equilibrium of the system (2) is $E_{0}=\left(\frac{b(1-p) N}{\mu}, 0,0\right)$.

Let $H=(X, Y, S)$ the system (2) can be written as

$H^{\prime}=\mathrm{E}(x)-\mathrm{V}(x)$

$\mathrm{E}(x)=\left[\begin{array}{c}\left(\beta X S+\frac{\beta Y S}{1+\alpha Y}\right) \\ 0 \\ 0\end{array}\right]$

The Jacobian matrix of $\mathrm{F}(x)$ at the DFE is

$\mathrm{DE}\left(E_{0}\right)=\left[\begin{array}{ccc}\beta \frac{b N}{\mu}(1-p) & \beta \frac{b N}{\mu}(1-p) & 0 \\ 0 & 0 & 0 \\ 0 & 0 & 0\end{array}\right]=\left[\begin{array}{cc}F & 0 \\ 0 & 0\end{array}\right]$

where $F=\left[\begin{array}{cc}\beta \frac{b N}{\mu}(1-p) & \beta \frac{b N}{\mu}(1-p) \\ 0 & 0\end{array}\right]$.

$V(x)=\left[\begin{array}{c}(\theta+\mu) X \\ -\theta X+(\delta+\gamma+\mu) Y \\ -b(1-p) N+\beta X S+\frac{\beta Y S}{1+\alpha Y}+\mu S\end{array}\right]$

The jacobian of $V(x)$ at the DFE is

$\operatorname{DV}\left(E_{0}\right)=\left[\begin{array}{ccc}\theta+\mu & 0 & 0 \\ -\theta & \delta+\gamma+\mu & 0 \\ \beta \frac{b N}{\mu}(1-p) & \beta \frac{b N}{\mu}(1-p) & \mu\end{array}\right]=\left[\begin{array}{ll}V & 0 \\ J_{1} & J_{2}\end{array}\right]$,

where $V=\left[\begin{array}{cc}\theta+\mu & 0 \\ -\theta & \delta+\gamma+\mu\end{array}\right]$

$V^{-1}=\left[\begin{array}{cc}\frac{1}{\theta+\mu} & 0 \\ \frac{\theta}{(\theta+\mu)(\delta+\gamma+\mu)} & \frac{1}{\delta+\gamma+\mu}\end{array}\right]$

The next generation matrix of the system (2) is:

$$
F V^{-1}=\left[\begin{array}{cc}
\frac{\beta b N}{\mu(\theta+\mu)}(1-p)+\frac{\beta b N \theta}{\mu(\theta+\mu)(\delta+\gamma+\mu)}(1-p) & \frac{\beta b N}{\mu(\delta+\gamma+\mu)}(1-p) \\
0
\end{array}\right]
$$

The spectral radius of the next generation matrix $\left(\rho F V^{-1}\right)$ is

$\frac{\beta b N((\delta+\gamma+\mu)+\theta)}{\mu(\theta+\mu)(\delta+\gamma+\mu)}(1-p)$.

Therefore $R_{0}=\frac{\beta b N((\delta+\gamma+\mu)+\theta)}{\mu(\theta+\mu)(\delta+\gamma+\mu)}(1-p)$

\section{Local Stability Of Disease Free Equilibrium State}

In epidemiology, if a system is stable under any condition(s), then the disease can be eradicated under such condition(s) in finite time. We now proceed to find the stability of the disease free equilibrium state.

The Jacobian matrix associated with the system (2) is: 


$$
J=\left[\begin{array}{ccc}
-\left(\beta X+\frac{\beta Y}{1+\alpha Y}+\mu\right) & -\beta S & \frac{-\beta S}{(1+\alpha Y)^{2}} \\
\left(\beta X+\frac{\beta Y}{1+\alpha Y}\right) & \beta S-(\theta+\mu) & \frac{\beta S}{(1+\alpha Y)^{2}} \\
0 & \theta & -(\delta+\gamma+\mu)
\end{array}\right]
$$

At the disease free equilibrium when there are no infectives we have,

$J\left(E_{0}\right)=\left[\begin{array}{ccc}-\mu & -\beta S^{*} & -\beta S^{*} \\ 0 & \beta S^{*}-(\theta+\mu) & \beta S^{*} \\ 0 & \theta & -(\delta+\gamma+\mu)\end{array}\right]$,

where $S^{*}=\frac{b N}{\mu}(1-p)$

The matrix $J\left(E_{0}\right)$ is stable if the following conditions hold

1) $\beta S^{*}-(\theta+\mu)-(\delta+\gamma+\mu)<0$

2) $\left(\beta S^{*}-(\theta+\mu)\right)(-(\delta+\gamma+\mu))-\theta \beta S^{*}>0$

From (4) we have,

$\frac{\beta b N(1-p)}{\mu(2 \mu+\theta+\delta+\gamma)}<1$

From(5)

$1>\frac{\beta b N((\delta+\gamma+\mu)+\theta)}{\mu(\theta+\mu)(\delta+\gamma+\mu)}(1-p)$

$\Rightarrow \frac{\beta b N((\delta+\gamma+\mu)+\theta)}{\mu(\theta+\mu)(\delta+\gamma+\mu)}(1-p)<1$

Or

$$
R_{0}<1 \text {. }
$$

But $R_{0}<1$ implies that the inequality (6) also holds since,

$$
\frac{\beta b N(1-p)}{\mu(2 \mu+\theta+\delta+\gamma)}<\frac{\beta b N((\delta+\gamma+\mu)+\theta)}{\mu(\theta+\mu)(\delta+\gamma+\mu)}(1-p)=R_{0} \text {. }
$$

Thus we have proved the following theorem

\section{THEOREM 1}

The system (2) is locally asymptotically stable if $R_{0}<1$

\subsection{GLOBAL STABILITY OF THE DISEASE FREE EQUILIBRIUM}

The global asymptotic stability of the disease free state of the model is investigated using the approach by Castillo-Chavevet al [13]. First the model is rewritten as

$$
\begin{aligned}
& \frac{d W}{d t}=H(W, Z), \\
& \frac{d Z}{d t}=G(W, Z), G(W, 0)=0
\end{aligned}
$$

where $W=(S)$ and $Z=(X, Y)$, with the components of $W \in \mathbb{R}$ denoting the uninfected population and the component of $Z \in \mathbb{R}^{2}$ denoting the infected population. $E_{0}=\left(W^{*}, 0\right)$ represent the disease-free equilibrium of the system.

The conditions for global stability are

i. $\quad \frac{d W}{d t}=H(W, 0), W^{*}$ is globally asymptotically stable (GAS)

ii. $\quad G(W, Z)=A Z-\hat{\mathrm{G}}(W, Z), \hat{\mathrm{G}}(W, Z) \geq 0$ for $(W, Z) \in \Gamma$

where $A=D_{z} G\left(W^{*}, 0\right)$ is an M-matrix (the off diagonal elements of $\mathrm{A}$ are non-negative) and $\Gamma$ is the region where the model makes biological sense.

If the system (2) satisfies the two conditions above then according to Castillo-Chavezet al. [Ref], the following theorem holds.

\section{THEOREM 2}

The equilibrium point $E_{0}=\left(W^{*}, 0\right)$ of system (2) is globally asymptotically stable provided $R_{0}<1$ (locally asymptotically stable) and that the conditions $(i)$ and (ii) are satisfied.

To apply theorem (2) on the system (2) we now proceed thus:

$$
\begin{gathered}
W=S(t), \quad Z=(X, Y), F(W, 0)=(b N(1-p)-\mu S) \\
A=D_{z} G\left(W^{*}, 0\right)=\left(\begin{array}{cc}
\beta S^{*}-(\theta+\mu) & \beta S^{*} \\
\theta & -(\delta+\gamma+\mu)
\end{array}\right)
\end{gathered}
$$


and

$$
\hat{\mathrm{G}}(W, Z)=\left(\begin{array}{c}
\hat{\mathrm{G}}_{1}(W, Z) \\
\hat{\mathrm{G}}_{2}(W, Z)
\end{array}\right)=\left(\begin{array}{c}
\beta X\left(S^{*}-S\right)+\beta Y\left(S^{*}-\frac{S}{1+\alpha Y}\right) \\
0
\end{array}\right)
$$

Since $S^{*}>S$ and $S^{*}>\frac{S}{1+\alpha Y}$, it is clear that $\hat{\mathrm{G}}(W, Z) \geq 0$. Therefore condition (ii) of theorem (2) is satisfied. Hence the disease free equilibrium may is globally asymptotically stable (GAS) when $R_{0}<1$.

\section{Existence Of Endemic Equilibria}

We now find the equilibria where at least one of the infected components of the system (2) is greater than zero.

Let $E_{1}=\left(S^{* *}, X^{* *}, Y^{* *}\right)$ denote the endemic equilibrium of the system (2), further

Let $\lambda^{* *}=\beta X^{* *}+\frac{\beta X^{* *}}{1+\alpha Y^{* *}}$

be the force of infection then at steady state

$S^{* *}=\frac{G}{\lambda^{* *}+\mu}$,

$X^{* *}=\frac{\lambda^{* *} S^{* *}}{A}$

and $Y^{* *}=\frac{\theta \lambda^{* *} S^{* *}}{A B}$.

Where $G=b N(1-p), A=\theta+\mu$

and $B=\delta+\gamma+\mu$.

Substituting (9) and (10)into (7) we have

$$
\begin{gathered}
\lambda^{* *}=\beta \frac{\lambda^{* *} S^{* *}}{A}+\frac{\beta \frac{\theta \lambda^{* *} S^{* *}}{A B}}{\frac{A B+\alpha \theta \lambda^{* *} S^{* *}}{A B}} \\
\lambda^{* *}=\beta \frac{\lambda^{* *} S^{* *}}{A}+\beta \frac{\theta \lambda^{* *} S^{* *}}{A B+\alpha \theta \lambda^{* *} S^{* *}} \\
1=\frac{\beta S^{* *}}{A}+\frac{\beta \theta S^{* *}}{A B+\alpha \theta \lambda^{* *} S^{* *}} \\
A\left(A B+\alpha \theta \lambda^{* *} S^{* *}\right)=\left(A B+\alpha \theta \lambda^{* *} S^{* *}\right) \beta S^{* *}+A \beta \theta S^{* *}
\end{gathered}
$$

Substituting (8) into (11) we have

$$
\begin{gathered}
A^{2} B+\frac{A \alpha \theta G \lambda^{* *}}{\lambda^{* *}+\mu}=\frac{\beta A B G}{\lambda^{* *}+\mu}+\frac{\alpha \beta \theta \lambda^{* *} G^{2}}{\left(\lambda^{* *}+\mu\right)^{2}}+\frac{A \beta \theta G}{\lambda^{* *}+\mu} \\
A^{2} B\left(\lambda^{* *}+\mu\right)+A \alpha \theta G \lambda^{* *}=\beta A B G+\frac{\alpha \beta \theta G^{2} \lambda^{* *}}{\lambda^{* *}+\mu}+A \beta \theta G \\
A^{2} B \lambda^{* *}+A^{2} B \mu+A \alpha \theta G \lambda^{* *}-\beta A G(\theta+B)-\frac{\alpha \beta \theta G^{2} \lambda^{* *}}{\lambda^{* *}+\mu}=0 \\
\left(A^{2} B-\frac{\alpha \beta \theta G^{2}}{\lambda^{* *}+\mu}+A \alpha \theta G\right) \frac{\lambda^{* *}}{A^{2} B \mu}+\left(1-R_{0}\right)=0 \\
\left(\left(\lambda^{* *}+\mu\right) A^{2} B-\alpha \beta \theta G^{2}+\left(\lambda^{* *}+\mu\right) A \alpha \theta G\right) \frac{\lambda^{* *}}{A^{2} B \mu}+\left(\lambda^{* *}+\mu\right)\left(1-R_{0}\right)=0 \\
\frac{\lambda^{* * 2}}{\mu}+\frac{\alpha \theta G \lambda^{* * 2}}{A B \mu}-\frac{\alpha \beta \theta G^{2} \lambda^{* *}}{A^{2} B \mu}+\frac{\alpha \theta G \lambda^{* *}}{A B}+2 \lambda^{* *}-R_{0} \lambda^{* *}+\left(1-R_{0}\right) \mu=0
\end{gathered}
$$

Equation (12) is in the following quadratic form

$$
a_{0} \lambda^{* * 2}+b_{0} \lambda^{* *}+c_{0}=0
$$

where $a_{0}=\frac{1}{\mu}+\frac{\alpha \theta G}{A B \mu}$

$b_{0}=2+\frac{\alpha \theta G}{A B}-\frac{\alpha \beta \theta G^{2}}{A^{2} B \mu}-R_{0}$ and

$$
c_{0}=\left(1-R_{0}\right) \mu
$$


The endemic equilibria can be obtained by solving for the positive values of $\lambda^{* *}$ in the quadratic (13) and substituting the results into (8),(9) and (10). Note that $a_{0}$ in (13) is always positive and $c_{0}$ is positive or negative if $R_{0}$ is less than unity or greater than unity respectively. We have therefore established the following.

\section{THEOREM3}

The system (2) has:

(a) precisely one unique endemic equilibrium if $c_{0}<0$ (i.e., $\left.R_{0}>1\right)$,

(b) precisely one unique endemic equilibrium if $b_{0}<0$, and $c_{0}=0$ (i.e., $R_{0}=1$ ) or $b_{0}^{2}-4 a_{0} c_{0}=0$,

(c) precisely two endemic equilibria if $c_{0}>0$ (i.e., $R_{0}<1$ ), $b_{0}<0$ and $b_{0}^{2}-4 a_{0} c_{0}>0$,

(d) no endemic equilibrium otherwise.

See $[14,15]$ for a similar approach.Case (a) of theorem 3 guarantees the existence of an endemic equilibrium whenever $R_{0}>1$. When $R_{0}<1$, case (c) indicates the possibility of a backward bifurcation (i.e., a situation where the locally asymptotically stable Disease Free Equilibrium co - exist with the locally asymptotically stable endemic equilibrium). This can be seen if we set the discriminant $b_{0}^{2}-4 a_{0} c_{0}=0$ and solve for a critical value of $R_{0}$. We get

$R_{0}^{c}=1-\frac{b_{0}^{2}}{4 a_{0} \mu}$

From (14) it can be shown that a backward bifurcation occurs for values of $R_{0}^{c}$ lying in the range $R_{0}^{c}<R_{0}<1$. Thus if $R_{0}^{c}<R_{0}<1$, then the system (2) exhibit a backward bifurcation.

\section{Bifurcation analysis}

We shall analyse the existence and stability of endemic equilibrium near the bifurcation point $x=x^{*}$ and $R_{0}=$ 1. The bifurcation analysis is performed at the disease-free equilibrium by using Centre Manifold Theorem as presented in Castillo-Chavez and Song [16].

Theorem 4 (Castillo-Chavez and Song, 2004)

Consider the following system of ordinary differential equations with parameter $ø$ such that $\frac{d x}{d t}=f(x, \varnothing), f: \mathbb{R}^{n} \times \mathbb{R} \rightarrow \mathbb{R}$ and $f \in \mathbb{C}^{2}\left(\mathbb{R}^{n} \times \mathbb{R}\right)$.

Without loss of generality, it is assumed that zero is an equilibrium point of the system for all parameters (that is $f(0, \varnothing) \equiv 0$ forallø) and

1. $A=D_{x} f(0,0)=\left(\frac{\partial f_{i}}{\partial x_{j}}(0,0)\right)$ is the linearization matrix of the system around the equilibrium 0 with $\phi$ evaluated at 0 . Zero is a simple eigenvalue of $A$ and other eigenvalues of $A$ have negative real parts;

2. Matrix A has a non-negative right eigenvector $w$ and a left eigenvector $v$ corresponding to the zero eigenvalue.

Let $f_{k}$ be the $k^{\text {th }}$ component of $f$ and

$a=\sum_{k, i, j=1}^{n} v_{k} w_{i} w_{j} \frac{\partial^{2} f_{k}}{\partial x_{i} \partial x_{j}}(0,0)$,

$d=\sum_{k, i=1}^{n} v_{k} w_{i} \frac{\partial^{2} f_{k}}{\partial x_{i} \partial \varnothing}(0,0)$

Then the local dynamics of the system around the equilibrium point 0 is totally determine by the signs of $a$ and $b$

i. $\quad a>0, d>0$. When $\varnothing<0$ with $|\varnothing| \ll 1,0$ is locally asymptotically stable and there exists a positive unstable equilibrium; when $0<ø \ll 1,0$ is unstable and there exist a negative, locally asymptotically stable equilibrium.

ii. $\quad a<0, d<0$. When $\varnothing<0$ with $|\varnothing| \ll 1,0$ is unstable; when $0<ø \ll 1,0$ is locally asymptotically stable equilibrium, and there exist a positive unstable equilibrium.

iii. $\quad a>0, d<0$. When $ø<0$ with $|\varnothing| \ll 1,0$ is unstable, and there exists a locally asymptotically stable negative equilibrium; when $0<ø \ll 1,0$ is stable and a positive unstable equilibrium appears.

$i v . \quad a<0, d>0$.when changes from negative to positive, 0 changes its stability from stable to unstable. Correspondingly a negative unstable equilibrium becomes positive and locally asymptotically stable.

Particularly if $a>0$ andd $>0$, then a subcrtical (or backward) bifurcation occurs at $\varnothing=0$.

To apply the theorem, we make the following change of variables

$x_{1}=S, x_{2}=X$ and $x_{3}=Y$ with

$x^{\prime}=f(x)$

Where $x=\left(x_{1}, x_{2}, x_{3}\right)^{T}$ and $f=\left(f_{1}, f_{2}, f_{3}\right)^{T}$ 
In non-compact form, equation (15) can be rewritten as thus:

$x_{1}^{\prime}=b(1-p) N-\beta x_{1} x_{2}-\frac{\beta x_{3} x_{1}}{1+\alpha x_{3}}-\mu x_{1}$

$x_{2}^{\prime}=\beta x_{1} x_{2}+\frac{\beta x_{3} x_{1}}{1+\alpha x_{3}}-(\theta+\mu) x_{2}$

$x_{3^{\prime}}=\theta x_{2}-(\delta+\gamma+\mu) x_{3}$

The basic reproduction number was computed to be given by

If $\beta=\beta^{*}$ is a bifurcation parameter such that $R_{0}=1$, then

$$
R_{0}=\frac{\beta b N((\delta+\gamma+\mu)+\theta)}{\mu(\theta+\mu)(\delta+\gamma+\mu)}(1-p)
$$

$$
\beta=\beta^{*}=\frac{\mu(\theta+\mu)(\delta+\gamma+\mu)}{\beta b(1-p) N((\delta+\gamma+\mu)+\theta)}
$$

For the computations of $\mathrm{a}$ and $\mathrm{b}$, the second partial derivatives of $\mathrm{f}$ at DFE are given by

$$
\frac{\partial^{2} f_{1}}{\partial x_{1} \partial x_{2}}=\frac{\partial^{2} f_{1}}{\partial x_{2} \partial x_{1}}=-\beta, \frac{\partial^{2} f_{1}}{\partial x_{1} \partial x_{3}}=\frac{\partial^{2} f_{1}}{\partial x_{3} \partial x_{1}}=-\beta
$$

$\frac{\partial^{2} f_{2}}{\partial x_{1} \partial x_{2}}=\frac{\partial^{2} f_{2}}{\partial x_{2} \partial x_{1}}=\beta, \frac{\partial^{2} f_{2}}{\partial x_{1} \partial x_{3}}=\frac{\partial^{2} f_{2}}{\partial x_{3} \partial x_{1}}=\beta$

$\frac{\partial^{2} f_{3}}{\partial x_{i} \partial x_{j}}=0, i, j=1,2,3$

We obtain

$a=v_{1} \sum_{i, j=1}^{3} w_{i} w_{j} \frac{\partial^{2} f_{1}}{\partial x_{i} \partial x_{j}}+v_{2} \sum_{i, j=1}^{3} w_{i} w_{j} \frac{\partial^{2} f_{2}}{\partial x_{i} \partial x_{j}}+v_{3} \sum_{i, j=1}^{3} w_{i} w_{j} \frac{\partial^{2} f_{3}}{\partial x_{i} \partial x_{j}}$

$a=-2 v_{1}\left(w_{1} w_{2}+w_{1} w_{3}\right) \beta+2 v_{2}\left(w_{1} w_{2}+w_{1} w_{3}\right) \beta$

$a=-2 \beta\left(w_{1} w_{2}+w_{1} w_{3}\right)\left(v_{2}-v_{1}\right)$, since $w_{1} w_{2}=w_{2} w_{1}, w_{1} w_{3}=w_{3} w_{1}$

We shall now calculate the values of $v_{1}, v_{2}, v_{3}, w_{1}, w_{2}, w_{3}$ to know the sign of a. solving for the left nullvector $v$ corresponding to the zero eigenvalue, $v D_{x} f\left(x^{*}, 0\right)=0$, when $R_{0}=1$, we obtain

$v_{1}=0$ and $v_{2}=\frac{(\delta+\gamma+\mu)}{\beta x_{1}{ }^{*}} v_{3}$

Then the left nullvector is given by

$v=\left(v_{1}, v_{2}, v_{3}\right)=\left(0, \frac{(\delta+\gamma+\mu)}{\beta x_{1}{ }^{*}} v_{3}, v_{3}\right)$

Where $v_{3}>0$ is a free left eigenvector.

For the right nullvector, $D_{x} f\left(x^{*}, 0\right) w=0$, we obtain

$w=\left(\begin{array}{c}w_{1} \\ w_{2} \\ w_{3}\end{array}\right)=\left(\begin{array}{c}\frac{\beta x_{1}{ }^{*}}{\mu}\left(w_{3}-w_{2}\right) \\ \left(\frac{\theta+\mu}{\theta}\right) w_{3} \\ w_{3}\end{array}\right)=w_{3}\left(\begin{array}{c}-\frac{\beta x_{1}{ }^{*}}{\mu} \\ \left(1+\frac{\mu}{\theta}\right) \\ 1\end{array}\right)$

For which $w_{3}>0$ is a free right eigenvector.

Since $v_{1}=0$

$a=\frac{-2 \mu \beta}{\theta^{2}}(\delta+\gamma+\mu) v_{3} w_{3}^{2}<0$

For the sign of $d$, it can be shown that the second partial derivatives of $\mathrm{f}$ at DFE are given by

$\frac{\partial^{2} f_{1}}{\partial x_{2} \partial \beta^{*}}=\frac{\partial^{2} f_{1}}{\partial x_{3} \partial \beta^{*}}=-x_{1}{ }^{*}, \frac{\partial^{2} f_{2}}{\partial x_{2} \partial \beta^{*}}=\frac{\partial^{2} f_{2}}{\partial x_{3} \partial \beta^{*}}=x_{1}{ }^{*}$

Then

$d=v_{2}\left(w_{2} \frac{\partial^{2} f_{2}}{\partial x_{2} \partial \beta^{*}}+w_{3} \frac{\partial^{2} f_{2}}{\partial x_{3} \partial \beta^{*}}\right)$

$d=v_{2}\left(w_{2}+w_{3}\right) x_{1}{ }^{*}$

Or 
$d=\left(\frac{\delta+\gamma+\mu}{\beta}\right)\left(\frac{\theta+\mu}{\theta}\right) v_{3} w_{3}>0$

Since $a<0$ and $d>0$, by theorem (4), the of the direction of the model(16) is supercritical (forward).

\section{HERD IMMUNITY}

Not all susceptibles in a population need to be vaccinated against a disease before the disease can be eliminated. There is a threshold level above which an infectious disease will cease to persist. If this level can be exceeded by vaccination then the disease can be eliminated without necessarily vaccinating the entire population. The concept is known as herd immunity and it was instrumental in the elimination of smallpox.

Let $p_{c}$ be the proportion of those vaccinated against the disease, then

$$
\begin{gathered}
R_{0}\left(1-p_{c}\right)=1 \\
\left(1-p_{c}\right)=\frac{1}{R_{0}} \\
p_{c}=1-\frac{1}{R_{0}}
\end{gathered}
$$

We define $p_{c}$ as the critical vaccination threshold. If the proportion of those vaccinated exceeds $p_{c}$, then the disease will die out. That is, there is not enough susceptible to trigger an outbreak even if infectives are introduced.

The basic reproduction number for measles is widely accepted as 18 thus

$$
p_{c}=1-\frac{1}{18}=0.9444 \simeq 0.94
$$

This implies that a minimum of $94 \%$ of the susceptible population must be vaccinated before measles can be eliminated from any region.

If however the proportion $p_{c}$ was not attained in a vaccination campaign but rather $p_{1}<p_{c}$ was attained then the balance of the infection might be disturbed without elimination per se. This often causes unforeseen problems such as the mean age of the infection being raised and given a false idea of elimination.

\section{Sensitivity Analysis}

Most of the analyses of epidemiological modeling are dependent on the Basic Reproduction number $R_{0}$ and the following sensitivity analysis is no exception. The magnitude of the Basic Reproduction number determine the level of the infection thus If $R_{0}$ is very sensitive to a particular parameter, then a perturbation of the conditions that connect the dynamics to such a parameter may prove useful in identifying policies or intervention strategies that reduce epidemic prevalence. Latin Hypercube Sampling (LHS) is a sophisticated and efficient statistical analysis technique that allows for simultaneous variation of the values of all input parameters [17]. In conjunction with LHS we used the Partial Rank Correlation Coefficient to perform the analysis of parameters (PRCC). PRCC vary between 1 and -1 and can be applied to any nonlinear monotonic relationship. PRCCs rank each parameter by the effect it has on the outcome when all other parameters are kept at median values. We used 100000 simulations per run.

Figure 4 illustrates the degree of sensitivity of each parameter on $R_{0}$, using ranges in the table. Parameters with PRCCs $>0$ will increase $R_{0}$ when they are increased, while parameters with PRCCs $<0$ will decrease $R_{0}$ when they are increased.Although carefully selected our parameter values are for illustration purposes only and may not represent actual field data.

Table 1: Parameter values used in PRCC

\begin{tabular}{lll}
\hline Symbol & Parameter & Range \\
\hline $\mathrm{N}$ & Total human population & $4000-10000$ \\
$\beta$ & Probability of infection upon contact & $0.08-0.7$ \\
$b$ & Birth rate & $0.1-0.3$ \\
$p$ & Individuals successfully vaccinated against the disease & $0.1-0.9$ \\
$\mu$ & Natural death rate & $0.01-0.6$ \\
$\theta$ & Rate at which the asymptomatically infectious become & $0.6-1$ \\
& symptomatic & $0.1-0.9$ \\
$\gamma$ & Recovery rate & $0.02-0.7$ \\
$\delta$ & Disease induced death rate & \\
\hline
\end{tabular}




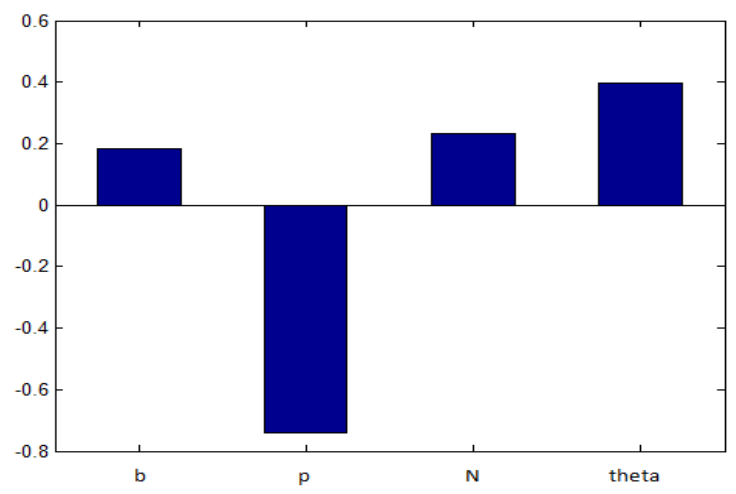

Fig 2: Sesnsitivity of $R_{0}$ to selected parameters using PRCC

Result of the PRCC (figure 2) shows that increasing the parameter $p$ will decrease the value of $R_{0}$ while increasing any of $b, N$ and $\theta$ will increase the value of $R_{0}$.

\section{Discussion}

Measles is a highly contagious disease with a greater than $90 \%$ attack rate. The disease is capable of taking over an entire susceptible community. Two important factors contribute to the high degree infectiousness of measles.

1. The disease is air-borne.

2. It is capable of being transmitted even before symptoms appear.

A single case of measles is usually considered an outbreak because of a very high basic reproduction number which makes the disease free equilibrium very unstable. Despite the availability of the measles vaccine since the past fifty years, measles remain both an endemic and an epidemic disease in many parts of the world especially in the developing nations. The France incidence is a pointer to the fact that the issue of herd immunity cannot be completely relied upon in the eradication or even elimination of measles as it creates a susceptible pool upon which the disease can thrive if introduced given its high reproduction number.

Due to poor vaccine uptake (Mostly as a result of the controversy surrounding the MMR vaccine at the time) measles was declared endemic in the UK in 2008 [18] and the disease continue to persist in the region. Between November 2012 and April 2013, 886 cases of the disease were reported following an outbreak centered on the Swansea area of Wales. The foregoing leads one to conclude that measles is a hard nut to crack in terms of elimination.

If a developing nation like Nigeria wishes to eliminate measles, then drastic actions needs to be taken. The herd immunity of $94 \%$ vaccine coverage is yet to be achieved in the country. According to WHO, Nigeria has about 1.7 million children who were not vaccinated against the disease in 2011 [19]. Even if 94\% vaccine coverage was to be achieved, several studies have shown that measles epidemics can still occur in highly vaccinated population [20-22]. The U.S incidence suggests that no one should be left unvaccinated. Measles was declared eliminated from the U.S in 2000. After 2000, prior to 200860 to 65 cases were imported into the U.S. each year but there is little or no secondary transmission. By 2008 and after the imported cases resulted in secondary transmission because there is a critical number of unvaccinated children.

\section{Conclusion}

We developed a mathematical model for measles epidemic. The basic reproduction number was found using the next generation matrix. The system was also found to be stable if $R_{0}<1$.This means that the disease can be eradicated or eliminated from a region under such condition in finite time. Measles have proven to be a difficult disease to eliminate as developed nations like UK and France are still faced with the challenge. If elimination is the target then a developing nation like Nigeria will require a more stringent effort. We note that:

$R_{0}=0<1$ given $p=1$ or

$\lim _{p \rightarrow 1} R_{0}=0$.

That is, the disease will certainly be eliminated if all susceptible are vaccinated. Achieving a $100 \%$ vaccination coverage is impractical but if the goal is set to $100 \%$ then we just might hit the $\geq 94 \%$ vaccine coverage which is the herd immunity for measles. Since measles is predominantly found among children aged 5 years and below, we therefore suggest that the measles vaccine should be made compulsory such that no child is allowed to enter school without evidence of at least two dose measles vaccination. 


\section{Refferences}

[1] Wikipedia.MMR vaccine controversy. Available fromhttp://en.wikipedia.org/wiki/MMR vaccine controversy. Accessed $5^{\text {th }}$ may 2013.

[2] U. Nnebe-Agumadu, Measles Control in Nigeria: The case for two dose vaccine policy. Nigerian Journal of Paediatrics, 32(3) 41 45, 2005.

[3] Public Health agency of Canada. Measles.Available from http://www.phac-aspc.gc.ca/im/vpd-mev/measles-rougeoleeng.php.Accessedon $5^{\text {th }}$ may 2013.

[4] World Health organization. Measles fact sheet.Available from http://www.who.int/mediacentre/factsheets/fs $286 /$ en/.Accessedon $5^{\text {th }}$ May 2013.

[5] R.F. Grais, M.J. Ferrari, C. Dubray, O.N. Bjørnstad, B.T. Grenfell, A. Djibo, F. Fermon, P.J. Guerin, Estimating transmission intensity for a measles epidemic in Niamey, Niger: lessons for intervention, Royal society of tropical medicine and hygiene, 100, $867-873,2006$

[6] E.A. Bakare,Y.A. Adekunle, K.O Kadiri, Modelling and Simulation of the Dynamics ofthe Transmission of Measles, International Journal of Computer Trends and Technology, 3(1), 2012.

[7] K.Ejima, R. Omori, K. Aihara, H. Nishiura, Real-time Investigation of Measles Epidemics with Estimate of Vaccine Efficacy, International Journal of Biological Sciences, 8(5) 620-629, 2012.

[8] J. Sachs,End of poverty; economic possibilities of our time. NY: Penguin Press, New York, 2005.

[9] M.H. Bonds, P. Rohani, Herd immunity acquired indirectly from interactions betweenthe ecology of infectious diseases, demography and economics, J. R. Soc. Interface 7, 541-547, 2010.

[10] Center for Disease Control and Prevention. Yellow book.Measles (Rubeola).Available from http://wwwnc.cdc.gov/travel/yellowbook/2012/chapter-3-infectious-diseases-related-to-travel/measles-rubeola. Accessed on $5^{\text {th }}$ may 2013

[11] O. Diekmann, J. A. P. Heesterbeek and M. G. Roberts, The construction of next-generation matrices for compartmental epidemic models, J. R. Soc. Interface, 2009 (doi:10.1098/rsif.2009.0386)

[12] P. Van den Driessche, J. Watmough, Reproduction numbers and sub-threshold endemic equilibria for compartmental models of disease transmission. Math.Biosci. 180, 29-48, 2002 doi:10.1016/S0025-5564(02)00108-6

[13] C. Castillo-Chavez, Z. Feng, and W. Huang, On the computation of Ro and its role on global stability,in: Castillo-Chavez C., Blower S., van den Driessche P., Krirschner D. and Yakubu A.A.(Eds), Mathematical Approaches for Emerging and Reemerging Infectious Diseases: An Introduction. The IMA Volumes in Mathematics and its Applications. Springer-Verlag, New York, 125(2002), pp. 229-250.

[14] S.M. Garba, A.B. Gumel and M.R. Abu Bakar, Backward Bifurcations in Dengue Transmission Dynamics, Mathematical Biosciences (2008), doi: 10.1016/j.mbs.2008.05.002

[15] Z. Mukandavire, A.B. Gumel, W. Garira and J.M. Tchuenche, Mathematical Analysis of a Model for HIV-Malaria CoInfection,Mathematical Biosciences And Engineering, 6(2), 333-362,2009 doi:10.3934/mbe.2009.6.333

[16] Castillo-Chavez, C. and B. Song "Dynamical models of tuberculosis and their applications", Mathematical Biosci. and Engr. Vol 1 No.2, pp. 361-404, 2004.Blower, S. M., \&Dowlatabadi, H. (1994). Sensitivity and uncertainty analysis of complex models of disease transmission: an HIV model, as an example. Int. Stat. Rev., 2, 229-243.

[17] S. M. Blower, H. Dowlatabadi, Sensitivity and uncertainty analysis of complex models of disease transmission: an HIVmodel, as an example. International Statistical Review. 2(1994) 229-243.

[18] Wikipedia the free encyclopedia,Measles outbreaks in the 21st century.Available from http://en.wikipedia.org/wiki/Measles outbreaks in the 21st century. Accessed on $10^{\text {th }}$ may 2013.

[19] Nigerian News World. Mealses rage in the north. Available from http://www.nigeriannewsworld.com/content/measles'-rage-north. Accessedon 15th may 2013.

[20] I.R. Pedersen, C.H. Mordhorst, G. Glikmann, et al. Subclinical measles infection in vaccinated seropositive individuals in arctic Greenland. Vaccine, 7:345-8, 1989.

[21] B.M. Nkowane, S.W. Bart, W.A. Orenstein, et al. Measles outbreak in a vaccinated school population: epidemiology, chains of transmission and the role of vaccine failures. Am. J. Public Health, 77:434-8. 1987.

[22] J. Mossong, D. J. Nokes, W. J. Edmunds. et al. Modeling the Impact of Subclinical Measles Transmission in Vaccinated Populations with Waning Immunity, American Journal of Epidemiology, 15(11), 1238 - 1249, 1999. 